\title{
Estudio de emisiones contaminantes utilizando combustibles locales
}

\section{Study of polluting emissions using local fuels}

\author{
EdD. Pérez Darquea Diego Gustavo \\ Universidad Internacional del Ecuador, Ecuador
}

Autor para correspondencia: dperez@uide.edu.ec

Fecha de recepción: 22 de Diciembre de 2017 - Fecha de aceptación: 01 de Marzo de 2018

Resumen: La tecnología en el control de emisiones de gases contaminantes se ha desarrollado notablemente en los últimos años ya que los efectos de estos residuos químicos son muy notables en el daño al ecosistema en general, ya que esto se ve reflejado en el calentamiento global y en el efecto invernadero, esto ha permitido que la industria automotriz se concentre en producir vehículos con bajo índice de emisiones para colaborar con el cuidado del medio ambiente, de la misma manera se han perfeccionado los sistemas para que cada vez sea inferior la contaminación en general, ya que de un sistema a carburación se desarrolló un sistema electrónico que redujo considerablemente las emisiones contaminantes. Cada vez se desarrolla más tecnología y la electrónica se impone más sobre ayudar al control de emisiones. El futuro en la industria automotriz para reducir la emisión de gases contaminantes son los vehículos híbridos. La ayuda de equipos sofisticados y especializados para determinar los valores exactos de emisiones de gases contaminantes cumplen un papel fundamental para ayudar al usuario o al técnico en controlar que estos gases no sobrepasen los valores establecidos por el organismo que controla dichos gases.

Palabras Clave: gases contaminantes; contaminación ambiental; control de emisiones

\begin{abstract}
The technology in the control of emissions of polluting gases has developed remarkably in the last years since the effects of these chemical residues are very remarkable in the damage to the ecosystem in general, since this is reflected in the global warming and in the Greenhouse effect, this has allowed the automotive industry to focus on producing vehicles with a low emissions index to collaborate with the care of the environment, in the same way the systems have been improved so that pollution in general is That from a carburizing system an electronic system was developed that considerably reduced pollutant emissions. More and more technology is being developed and electronics are more important to help with emissions control. The future in the automotive industry to reduce emissions of greenhouse gases are hybrid vehicles. The aid of sophisticated and specialized equipment to determine the exact values of emissions of polluting gases play a fundamental role in helping the user or the technician in controlling that these gases do not exceed the values established by the organism that controls those gases.
\end{abstract}

Key Words: contaminant gases; environmental pollution; emission control 


\section{Introducción}

En el presente artículo se realizó un estudio de comparación de los gases contaminantes en el Ecuador con combustibles locales los cuales son gasolina súper, extra y eco-páis para esto, es importante conocer cuáles son los gases expulsados por el motor después de la combustión, además saber las normativas contaminantes del país para comparar y tener datos cuantitativos acerca de cuál es el combustible que menos contamina.

En el país se utiliza 3 tipos de gasolinas: Extra, Súper y Eco-país. En el año 2012 se alcanzó la cifra de 23.6 millones de barriles en todo el país, de este total el $73 \%$ corresponde a gasolina Extra, el 22.5\% a gasolina Súper, y el 4,5 a Eco-País (Mena Paúl. (2010). Ecuador arranca venta de biocombustible. Junio 2017, de BBC Sitio web: http://www.bbc.com/mundo/ciencia_tecnologia/2010/01/100112_0245_ecuador_gasolina_ve rde_jrg)

La gasolina Eco-país es alcohol etílico anhídrido al 5\% en volumen en una mezcla con gasolina extra que se comercializaba como parte de un plan piloto en Guayaquil desde el año 2010. Actualmente hay la posibilidad para escalar ese plan piloto a nivel nacional debido a que el uso de biocombustibles es parte de las estrategias del gobierno actual.

Los gases contaminantes que expulsa el motor son: dióxido de azufre (SO2), dióxido de carbono (CO2), monóxido de carbono (CO) y óxido de nitrógeno (NOx). Las emisiones contaminantes de gases producidas por el motor de combustión interna son responsables únicamente de un $5 \%$ de las emisiones de dióxido de azufre (SO2), son responsables del $25 \%$ de las emisiones de dióxido de carbono (CO2), del $87 \%$ de las de monóxido de carbono (CO) y del $66 \%$ de las de óxidos de nitrógeno (NOx). (Ovando René. (2010). Los gases efecto invernadero. En contaminación atmosférica por bióxido de carbono emitido por vehículos automotores en la ciudad de Torreón (22 - 31). Coahuila - México: Universidad Antonio Narro.)

La principal característica de un combustible es su poder calorífico, que es el calor desprendido por la combustión completa de una unidad de masa de combustible. El calor o poder calorífico, También llamado capacidad calorífica, se mide en julio, caloría o BTU, dependiendo del sistema de unidades. Por todas estas razones se está intentado por todos los medios posibles la reducción de los gases de escape y sus emisiones contaminantes.

La principal fuente de emisión de $\mathrm{CO}_{2}$ es la combustión que se origina en el uso de combustibles fósiles esto es gas carbón y petróleo; los cuales se utilizan en los procesos industriales, en el sector petrolero y en el transporte.

\section{Combustibles}

\section{Fundamento teórico}

La gasolina es un producto obtenido en la destilación fraccionada del petróleo crudo, esto significa que depende del crudo que se destile es una fracción única o una mezcla de diversas fracciones, está formada de una mezcla de hidrocarburos de peso molecular no muy elevado debe ser volátil, par que queme fácilmente y para mejorar el arranque en frio, pero no tanto como 
para formar demasiado vapor con el tiempo caluroso. (CCT. (2011). Introducción A La Refinación Del Petróleo Y Producción De Gasolina Y Diésel Con Contenido Ultra Bajo De Azufre. Marzo 2017, de $\pi$ Math Pro Sitio web:

https://www.theicct.org/sites/default/files/ICCT_RefiningTutorial_Spanish.pdf)

Las gasolinas empleadas en motores de combustión interna satisfacen diversos requerimientos tanto en su etapa de producción como es su posterior rendimiento vehicular, su composición química está formada por moléculas de carbono e hidrogeno normalmente tiene entre 7 y 11 átomos de carbono unidos a átomos de hidrogeno. En el petróleo los átomos de carbono se encuentran unidos por cadenas de diferentes longitudes por lo que las moléculas con distintas longitudes presentan propiedades y comportamientos diversos (Rodger W., Griffin Jr. (1981). Química orgánica moderna. Barcelona - España: REVERTÉ.) La gasolina está formada por hidrocarburos que pertenecen a la familia de los alcanos cuya configuración química es:

$$
\mathrm{Cr} H 2 r+2
$$

De este arreglo obtiene la siguiente lista de hidrocarburos, Los hidrocarburos son compuestos orgánicos formados únicamente por átomos de carbono e hidrógeno. La estructura molecular consiste en un armazón de átomos de carbono a los que se unen los átomos de hidrógeno.

1. $n=1 \quad C \quad H 4$ Metano

2. $n=2 \quad C 2 H 6 \quad$ Etano

3. $n=3 \quad \mathrm{C} 3 \mathrm{H} 8$ Propano

4. $n=4 \quad C 4 H 10$ Butano

5. $n=5 \quad$ C5 H12 Pentano

6. $n=6 \quad$ C6 H14 Exano

7. $n=7 \quad$ C7 H16 Heptano

8. $n=8 \quad$ C8 H18 Octano

Las primeras cuatro cadenas de alcanos (metano, etano, propano y butano) son gases, a partir de estas y hasta llegar al C18 son líquidos a temperatura ambiente y las cadenas después del C19 son sólidos a temperatura ambiente (Chow Susana. (1987). Petroquímica y Sociedad. México: 1987.) Las series de operaciones efectuadas en una refinería se producen muchos destilados crudos que son tratados químicamente antes de enviarse a los tanques finales de mezclado (Parra Enrique. (2003). Petróleo y gas natural. Madrid - España: AKAL S.A...)

\section{Propiedades físico - químicas}

En términos generales, las características que definen el comportamiento de un combustible en el motor, son el poder antidetonante y la volatilidad, los otros requerimientos limitan la presencia de componentes indeseables a concentraciones tan bajas, de tal manera que no tengan un efecto adverso en el funcionamiento de la máquina. 


\section{Octanaje}

El octanaje en la gasolina es la medida de su calidad antidetonante, es decir la habilidad que tiene para quemarse sin causar detonación en los motores de combustión interna. Para medir esta propiedad existen tres métodos: Numero de octano (M.O.N), Numero de octano Investigado (R.O.N) y Numero de octano en marcha. (Scott Fogler. (2001). Elementos de ingeniería de las reacciones químicas. España: PEARSON.)

El M.O.N es tomado como una indicación, la habilidad del combustible para evitar la detonación de motores en altas velocidades. El R.O.N mide la tendencia de detonación a bajas velocidades, mientras que el Número de octano en marcha es el que realmente muestra cómo se comporta el combustible en el motor del automóvil.

\section{Detonación}

Normalmente la chispa salta en la bujía cuando el pistón está cerca del P.M.S inflama la mezcla y los gases se propagan en todas direcciones principalmente hacia los extremos de la cámara de compresión, teniendo como punto de partida el de la inflamación inicial, así que la fuerza explosiva se aplica de modo rápido pero progresivo al pistón que a su vez se mueve hacia el P.M.I.

\section{Combustible Ecopaís}

El Ministerio Coordinador de Producción, Empleo y Competitividad (MCPEC), en el marco del Cambio de la Matriz Productiva, fomenta la producción de la gasolina ECOPAÍS, un biocombustible compuesto de 5\% de bioetanol (proveniente de la caña de azúcar) y un $95 \%$ de gasolina base. (Castillo P, Caballero P. (2012). Análisis de las propiedades fisicoquímicas de gasolina y diésel mexicanos reformulados con Etanol. Ingeniería Investigación y Tecnología, XIII, 14. 2017, De SCIELO Base de datos.)

ECOPAÍS posee el mismo octanaje (87 octanos) y precio de la gasolina Extra. Este proyecto empezó, como fase piloto, el 12 de enero de 2010 en Guayaquil. Para la producción de gasolina Extra con 87 octanos se requiere mezclar un $76 \%$ de nafta de alto octano (NAO), las cuales se importan, con $24 \%$ de naftas de bajo octano (NBO); mientras que para la producción de ECOPAÍS, con la misma cantidad de octanos, se requiere $62 \%$ de NAO, 33\% de NBO y un 5\% de bioetanol (Castillo P, Caballero P. (2012). Análisis de las propiedades fisicoquímicas de gasolina y diésel mexicanos reformulados con Etanol. Ingeniería Investigación y Tecnología, XIII, 14. 2017, De SCIELO Base de datos.)

Gracias al alto octanaje de bioetanol, al incorporarlo en la producción de ECOPAÍS, genera una reducción de la producción de NAO en un 14\%, lo que implica menos importaciones de este derivado y un efecto positivo en la balanza comercial nacional. En 2010 su habitual enlace sabatino el mandatario oficializó el decreto del ingreso de un nuevo tipo de combustible. Se trata de la denominada Ecopaís que tiene como objetivo principal reducir las emisiones de carbono al ambiente. 
A diferencia de la gasolina Extra corriente, Ecopaís integra un 5\% de etanol, una variante de combustible de origen orgánico que proviene del maíz y la caña de azúcar. En el caso de nuestro país se usa caña de azúcar. Desde el 2010 este tipo de combustible ya se comercializa en algunos sectores del litoral ecuatoriano, ahora el reto es que hasta el 2017 Ecopaís remplace en su totalidad el uso de Extra.

Para este propósito se ha establecido que necesitan un promedio de 30.000 hectáreas de suelo para el cultivo de caña, que a su vez generará nuevas fuentes de ingreso y alrededor de 9.000 puestos de trabajo teniendo como estandarte que este será un carburante $100 \%$ ecuatoriano.

Correa afirmó que la gasolina de etanol cumple con los mismos estándares de la Extra, en cuanto a potencia y precio, pero dijo que brinda otros beneficios como una mayor protección a los motores. Además, se ahorrarían cerca de 280 millones de dólares por concepto de subsidio al combustible en los siguientes 5 años. Se espera que una vez que se generalice el consumo de Ecopaís las emisiones de carbono al ambiente disminuirán considerablemente equivalentes a 80.000 vehículos. (García Christian. (2015). Gasolina Eco-país reemplazará a Extra hasta el 2017. ACELERANDO, 39, 46.)

Según la Agencia de Protección Ambiental de los Estados Unidos (EPA), conducir un automóvil es la actividad individual más contaminante que la mayoría de la gente realiza. (EPA. (1996). Manual de Participación: Publica de la RCRA (RCRA Public Participation Manual). Marzo 2017, de EPA Sitio web https://nepis.epa.gov/Exe/ZyPURL.cgi?Dockey=100 00Y1 Z. txt). Los vehículos a motor liberan millones de toneladas de contaminantes a la atmósfera cada año. En muchas zonas urbanas, los vehículos son los principales responsables de la presencia de ozono a nivel del suelo. Éste es uno de los componentes principales del smog y constituye el problema más grave en términos de polución del aire en los estados del noreste y los de la zona media de la Costa Este del país. Además, los automóviles emiten diversos contaminantes clasificados como tóxicos, los cuales son causantes de nada menos que 1.500 casos de cáncer en el país al año. Las emanaciones de los automóviles también son causantes de problemas medioambientales, tales como la lluvia ácida y el calentamiento global del planeta.

Las medidas para el control de la contaminación han permitido reducir drásticamente el nivel de emisiones por vehículo en los últimos veinte años. Sin embargo, durante dicho período el total de millas recorridas se ha duplicado, lo que ha dado lugar a un incremento del nivel de contaminantes del aire en muchos lugares del país.

\section{Contaminación}

Los vehículos emiten tres contaminantes principales: hidrocarburos, óxidos de nitrógeno y monóxido de carbono. Los hidrocarburos reaccionan con los óxidos de nitrógeno por acción de la luz solar y a temperaturas elevadas, formando ozono a nivel del suelo, el cual puede causar irritación de los ojos, tos, jadeo, insuficiencia respiratoria y trastornos pulmonares permanentes.

Los óxidos de nitrógeno (NOX) contribuyen a la formación de ozono y de lluvia ácida, además de afectar la calidad del agua. El monóxido de carbono es un gas incoloro letal que 
reduce el flujo de oxígeno en el torrente sanguíneo y puede afectar las funciones cerebrales y la visión.

En las zonas urbanas, los vehículos son responsables de nada menos que el 90 por ciento del monóxido de carbono del aire. Los vehículos a motor también emiten grandes cantidades de dióxido de carbono, el cual atrapa la radiación solar reflejada por la superficie terrestre, causando así el calentamiento global.

Los automóviles despiden contaminantes por el tubo de escape como consecuencia de la combustión de la gasolina y a través del capó y del sistema de combustible cuando el calor provoca la evaporación del mismo. La emisión por evaporación tiene lugar diferentes situaciones, como cuando la temperatura exterior en días cálidos y soleados provoca la evaporación de combustible; o cuando el calor del motor y del sistema de escape provoca un aumento de la temperatura del combustible; también si el auto tiene el motor apagado y permanece lo suficientemente caliente como para que el combustible se evapore, es importante saber que al cargar combustible, los vapores de la gasolina escapan del tanque a través del pico, lo que es recomendable cargar de combustible en las primeras horas de la mañana.

La mayor cantidad de contaminantes es emitida durante el "arranque en frío" o durante los minutos que tarda el automóvil en calentarse. (Suarez Víctor. (2007). Salud Ambiental De Los Estados Unidos. Santo Domingo). Dado que un auto se calienta más rápidamente cuando está circulando, se aconseja limitar el tiempo de calentamiento del motor. El aprovechar un mismo viaje para realizar más de una actividad contribuye a disminuir el nivel de emisiones, ya que reduce la cantidad de arranques en frío.

La oferta de vehículos de bajo nivel de emisiones, también llamados "autos californianos", irá en aumento durante los próximos años. (Suarez Víctor. (2007). Salud Ambiental De Los Estados Unidos. Santo Domingo). La diferencia entre estos vehículos y los tradicionales radica en que los primeros poseen un sistema adicional de control de emisiones.

Los gases emitidos por un motor de combustión interna de gasolina son, principalmente, de dos tipos: inofensivos y contaminantes. Los primeros están formados, fundamentalmente, por Nitrógeno, Oxígeno, Dióxido de Carbono, vapor de agua e Hidrógeno. Los segundos contaminantes están formados, fundamentalmente, por el Monóxido de Carbono, Hidrocarburos, Óxidos de Nitrógeno y Plomo.

\section{Gases inofensivos}

El Nitrógeno es un gas inerte que se encuentra presente en el aire que respiramos en una concentración del 79\%. Debido a las altas temperaturas existentes en el motor, el Nitrógeno se oxida formando pequeñas cantidades de Óxidos de Nitrógeno, aunque sea un gas inerte a temperatura ambiente. El Oxígeno es uno de los elementos indispensables para la combustión y se encuentra presente en el aire en una concentración del 21\%. (Martinez Fernado. (2014). Tipos de gases producidos en la combustión y sus consecuencias. Mayo 2917, de AS Catalizdores Sitio web: https://www.as-sl.com/pdf/tipos_gases.pdf) 
Si su mezcla es demasiado rica o demasiado pobre, el Oxígeno no podrá oxidar todos los enlaces de Hidrocarburos y será expulsado con el resto de los gases de escape. El vapor de agua se produce como consecuencia de la combustión, mediante la oxidación del Hidrógeno, y se libera junto con los gases de escape.

El Dióxido de Carbono producido por la combustión completa del Carbono no resulta nocivo para los seres vivos y constituye una fuente de alimentación para las plantas verdes, gracias a la fotosíntesis. Se produce como consecuencia lógica de la combustión, es decir, cuanto mayor es su concentración, mejor es la combustión. Sin embargo, un incremento desmesurado de la concentración de Dióxido de Carbono en la atmósfera puede producir variaciones climáticas a gran escala, llamado efecto invernadero.

\section{Materiales y métodos}

La metodología que se va a utilizar para esta investigación es sacar conclusiones mediante los experimentos, para que, de toda la información recolectada, salgan parámetros y datos reales de las consecuencias o beneficios que se pueden dar al usar gasolina extra con o sin aditivo.

Lugar

Según las condiciones geográficas el proyecto se realizó a 2800msnm, utilizando los equipos y el laboratorio a esa altura de trabajo. Las pruebas se darán en Quito a la altura antes mencionada, viendo cómo afecta a la emisión de gases factores como la altura, densidad del aire, etc.

\section{Vehículo}

El vehículo que se va a usar es un Grand Vitara SZ de la línea SUV, cuyo tipo es de los más vendidos en el ecuador, las marcas Chevrolet y kia son las marcas más cotizadas en el mercado ecuatoriano.

La idea es escoger Chevrolet porque tiene productos globales con diseño y tecnología con altos estándares y un elevado índice de clientes satisfechos. Por lo cual la marca Chevrolet se ha destacado por ser una de las marcas más vendidas en el mercado. Chevrolet es la marca más fuerte en Ecuador y en la región con importantes ventajas competitivas.

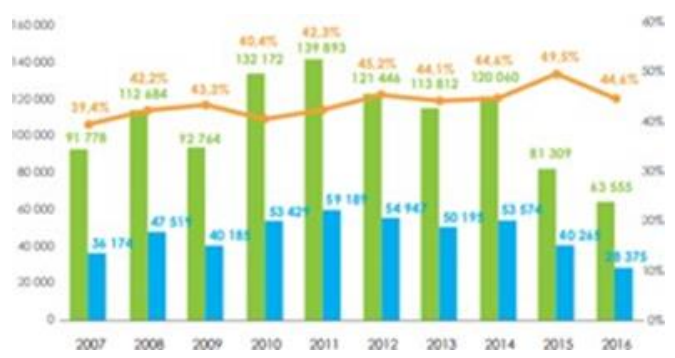

Figura 3. Ventas de vehículos Chevrolet en el Ecuador en los años 2007-2016

Fuente: El Telégrafo 


\section{Combustible}

El combustible por utilizar serán los distribuidos a nivel nacional, como es la gasolina extra, la gasolina súper y el eco-país. En noviembre de 2011 llegaron al ecuador las primeras importaciones de gasolina con 95 octanos, según Petroecuador. En las refinerías del país esta nueva gasolina fue mezclada y procesada con la que se tenía anteriormente. Por ello, el índice de octanaje mejoro en las gasolinas extra (de 81 a 87 octanos) y súper (de 90 a 92 octanos).

El octanaje determina la calidad y la capacidad de consumo en la gasolina. Una gasolina con mayor grado de octanos mejora la potencia y el rendimiento del motor, además disminuye el consumo de combustible. Por lo tanto, las gasolinas a usarse serán la extra y la súper porque resultan ser las más óptimas en el país.

\section{Equipo}

Cuando un analizador de gases, se usa en combinación con otros equipos de diagnóstico, se convierte en una de las más importantes herramientas para diagnosticar adecuadamente problemas relacionados con el funcionamiento del motor en general Un analizador de cuatro gases, está equipado con una bomba de vacío, que arrastra los gases de escape a través de una manguera de muestra insertada en el tubo de escape del automóvil y de ahí al analizador de gases, donde una muestra de gas de escape pasa al interior del analizador; una emisión de luz infrarroja es proyectada a través de la muestra de gas de escape.

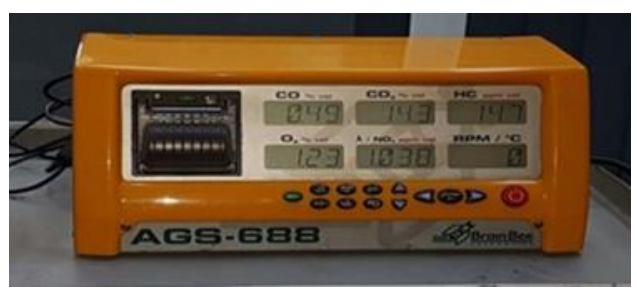

Figura 4. Analizador de gases

\section{Normativa}

Las normativas 2-203 y 2204 establecen referencias para el control de la emisión de gases. Esta norma establece el método de ensayo para determinar la concentración de las emisiones provenientes del sistema de escape de vehículos equipados con motor de encendido por chispa, en condiciones de marcha mínima o "ralenti". La norma se aplica a los vehículos automotores cuyo combustible es gasolina. Por otro lado, lo fundamental de estas normativas es que permiten regular y controlar a un nivel óptimo la contaminación emitidas por los vehículos. 


\section{Resultados y discusión}

En primera instancia se calibra los equipos a por lo que se las muestras fueron utilizadas en tubos de ensayo para las respectivas pruebas.
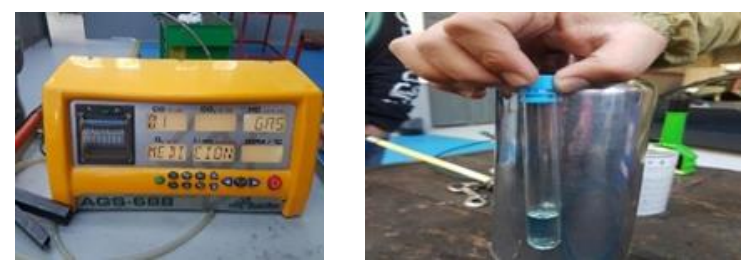

Figura 5. Materiales a utilizar

Luego de calentar el vehículo por aproximadamente 15 minutos se mide la emisión de gases contaminantes, alrededor de 5 muestras por combustible a estudiar.

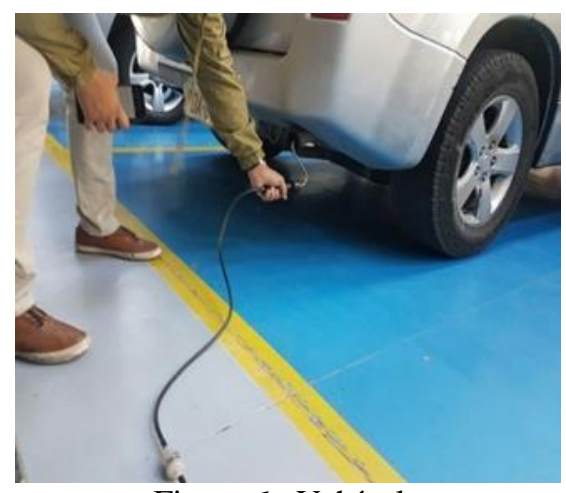

Figura 6. Vehículo

Utilizando una bomba de combustible externa cuya presión nominal está en las 30lb, se estandariza la presión de combustible de trabajo en todas las muestras para tener datos comparativos a tabular. Finalmente, con el analizador de gases, se verificó los datos obtenidos después de cada prueba según la normativa.

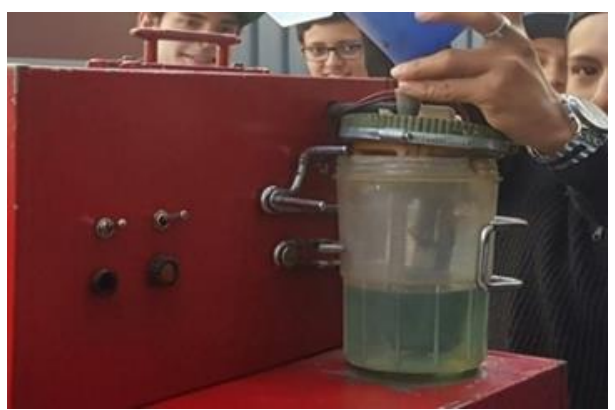

Figura 7. Bomba gasolina Externa

Las pruebas por realizar serán inicialmente con los combustibles origínales en el vehículo, teniendo la gasolina extra, súper, y el eco-país, sin colocar ningún aditivo o elemento que varíe en sus resultados. 


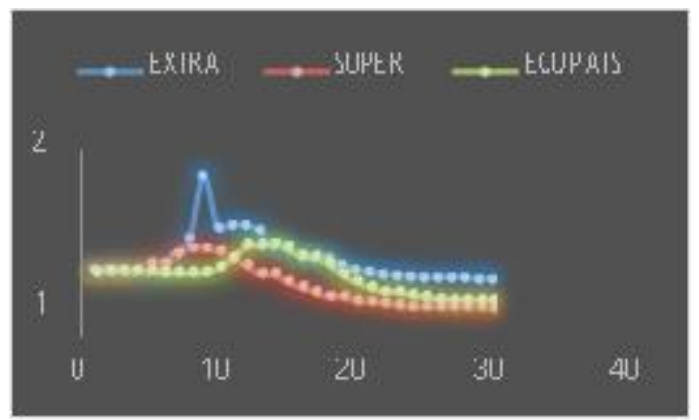

Figura 8. Porcentaje de CO Extra - Súper - Eco país

Las pruebas realizadas se las hicieron con los 3 combustibles teniendo como resultado final puntos máximos de emisiones de gases contaminantes según norma, cuyos valores en $\mathrm{CO}$, no deben pasar los 0,60 en \% de CO, pero como se ve en la gráfica existe un pico al momento de acelerar, teniendo una mezcla ligeramente rica, lo que eleva el valor de Co hasta 1,72 y luego es establece en 0,56 , con gasolina extra respectivamente y exista una disminución con los 2 combustibles a estudiar.

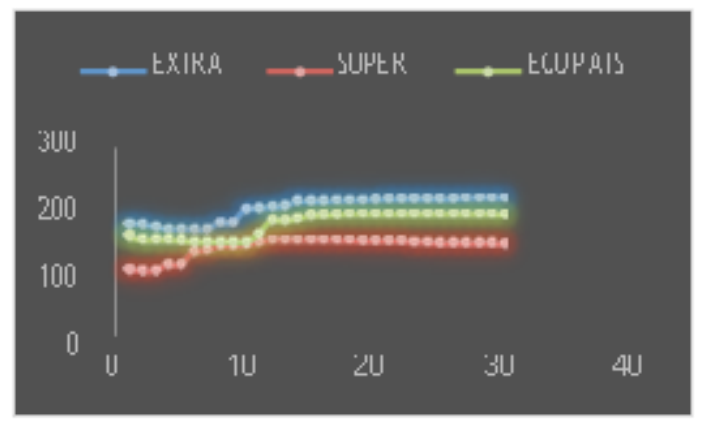

Figura 9. Porcentaje de HC Extra - Súper - Eco país

En el Ecuador se rige a la norma de INEN 2204 a temperatura normal de 0-1500 el porcentaje de CO no debe exceder de 1,0 y de 1500-3000 el porcentaje es de 1,0 (Subsecretaría de la Calidad del Ministerio de Industrias y Productividad. (2016). Norma INEN 2204. Julio 2017, de Gestión Ambiental Sitio web:

http://www.aeade.net/wpcontent/uploads/2016/12/PROYECTO-A2-NTE-INEN-2204.pdf). Considerando la altura, de $0-1500 \mathrm{msnm}$ el porcentaje de $\mathrm{HC}$ no debe exceder de $200 \mathrm{y}$ de 1500-3000 el porcentaje es de 200. La mezcla de prueba, la cual fue 0,01 ml de aditivo en $300 \mathrm{ml}$ de gasolina, se determinó realizando una regla de tres que nos permitió garantizar la prueba.

En las Figuras de la primera prueba (Gasolina extra), se puede observar que, a los 6 segundos los niveles de CO comienzan manteniéndose bajos con 0,7 hasta los 9 segundos donde alcanza un valor de 1,72. Inmediatamente ocurre una depreciación a los 10 segundos de 1,17, los cuales al finalizar la prueba en el segundo 30 se obtiene un dato de 0,62 , que está dentro de la norma INEN 2204. En el Figura de ppm HC se puede observar que a los 8 segundos los niveles de HC comienzan relativamente bajos con 175 ppm, manteniéndose bajo hasta los 11 segundos, donde alcanza las 198ppm. A partir de este momento, comienza un incremento de los valores, los cuales se elevan y llegan a los 235 ppm al finalizar la prueba en los 30 segundos.

En cuanto a los gases emitidos en la tercera prueba con el Eco-País parte de los siguientes valores: $\mathrm{CO} 0.68 \%$ vol, CO2 $14.3 \%$ vol, $\mathrm{HC} 159$ ppm vol, O2 0.85\% vol, $/$ /NOX 1.013, RPM/ 
C0 0. Los cuales dentro del rango de 0 a 30 segundos fueron variando de forma que oscilan (oscila como se ve en la gráfica) hasta llegar al tiempo límite y verificar valores con los valores ya determinados por las normas INEN. Los valores finales de la prueba son: $\mathrm{CO} 0.39 \%$ vol, $\mathrm{CO} 2$ 14.5\% vol, HC 194 ppm vol, O2 0.75\% vol, K/NOX 1.016, RPM/ C0 0. Así se evidencia que el auto de prueba estaba dentro del rango aceptado para circular.

\section{Conclusiones}

Realizando las pruebas, se evidencia de manera clara que los dos combustibles tienen comportamientos favorables según norma ya que los 2 de ellos bajan momentáneamente los niveles de emisiones de gases, pasando sin problema las revisiones vehiculares.

Es importante considerar las variables en las que se realiza las pruebas, ya que además del tipo de octanaje del combustible influye elementos como la altura, densidad del aire, humedad relativa, por lo que en base a estos parámetros los valores de emisiones de gases contaminantes que emite el combustible Extra en relación al Eco-País presenta valores similares en el porcentaje de $\mathrm{HC}$, mientras que al analizar el $\mathrm{CO}$, el combustible Eco-País presenta condiciones similares emisiones con la gasolina Súper obteniendo como resultado.

En las pruebas, se observa que la gasolina Súper actúa favorablemente, ya que los valores oscilan, pero de manera más favorable. El valor inicial de $\mathrm{CO}$ es 0,69; el final es 0,31. El único valor que sube de manera notable es en los 8 segundos donde emite un valor de 0,95 . En el Figura de ppm HC ocurre algo similar, donde el valor inicial es 106 y el valor final es 147. Se obtiene un valor un poco alto en relación al valor final, el cual se dio en el rango de 12 a 17 segundos.

\section{Bibliografía}

Castillo, P, \&Caballero, P. (2012). Análisis de las propiedades fisicoquímicas de gasolina y diésel mexicanos reformulados con Etanol. Ingeniería Investigación y Tecnología, XIII, 14. 2017, De SCIELO Base de datos

CCT. (2011). Introducción A La Refinación Del Petróleo Y Producción De Gasolina Y Diésel Con Contenido Ultra Bajo De Azufre. Marzo 2017, de $\pi$ Math Pro Sitio web: https://www.theicct.org/sites/default/files/ICCT_RefiningTutorial_Spanish.pdf

Chow, S. (1987). Petroquímica y Sociedad. Mexico: 1987.

EPA. (1996). Manual de Participacion: Publica de la RCRA (RCRA Public Participation Manual).

Marzo 2017, de EPA Sitio web https://nepis.epa.gov/Exe/ZyPURL.cgi?Dockey=100 00Y1 Z. txt

García, C. (2015). Gasolina Eco-país reemplazará a Extra hasta el 2017. ACELERANDO, 39, 46.

Martinez, F. (2014). Tipos de gases producidos en la combustión y sus consecuencias. Mayo 2917, de AS Catalizdores Sitio web: https://www.assl.com/pdf/tipos_gases.pdf 
Men, P. (2010). Ecuador arranca venta de biocombustible. Junio 2017, de BBC Sitio web: http://www.bbc.com/mundo/ciencia_tecnologia/2010/01/100112_0245_ecuador_gasolina _verde_jr g

Ovando, R. (2010). Los gases efecto ivernadero. En contaminación atmosférica por bióxido de carbono emitido por vehículos automotores en la ciudad de Torreón (22 - 31). Coahuila México: Universidad Antonio Narro.

Rodger W., \& Griffin Jr. (1981). Química orgánica moderna. Barcelona - España: REVERTÉ.

Scott Fogler. (2001). Elementos de ingeniería de las reacciones químicas. España: PEARSON.

Suarez, V. (2007). Salud Ambiental De Los Estados Unidos. Santo Domingo

Subsecretaría de la Calidad del Ministerio de Industrias y Productividad. (2016). Norma INEN 2204. Julio 2017, de Gestión Ambiental Sitio web: http://www.aeade.net/wpcontent/uploads/2016/12/PROYECTO-A2-NTE-INEN2204.pdf 